\title{
Sleep State Misperception or Paradoxical Insomnia?
}

\author{
Luciane Andrade Barreto ${ }^{1}$, João E Coin-Carvalho², Luciane BC Carvalho ${ }^{3}$, Lucila BF \\ Prado $^{4}$, Gilmar F Prado 5 \\ ${ }^{1}$ Psychologist, PhD Student, Neuro-Sono Sleep Center, Department of Neurology, Universidade Federal de São \\ Paulo, São Paulo, SP, Brazil. \\ ${ }^{2} \mathrm{PhD}$, Department of Psychology, Universidade Paulista and Neuro-Sono Sleep Center, Department of Neurology, \\ Universidade Federal de São Paulo, São Paulo, SP, Brazil. \\ ${ }^{3} \mathrm{PhD}$, Neuro-Sono Sleep Center, Department of Neurology, Universidade Federal de São Paulo, \\ São Paulo, SP, Brazil. \\ ${ }^{4} \mathrm{MD}$, PhD, Neuro-Sono Sleep Center, Department of Neurology, Universidade Federal de São Paulo, \\ São Paulo, SP, Brazil. \\ ${ }^{5} \mathrm{MD}$, PhD, Neuro-Sono Sleep Center, Department of Neurology, Universidade Federal de São Paulo, \\ São Paulo, SP, Brazil.
}

\begin{abstract}
:
Introduction: Sleep state misperception (SSM) intrigues sleep specialists as a disorder in which a complaint of insomnia is presented without any objective clinical evidence. International committees involved in producing sleep disorder classifications have included SSM in the insomnia group and classified this condition with a new name, paradoxical insomnia (PI).

Objective: The aim of this work is to discuss the adoption of the term "paradoxical insomnia" by the last international classification.

Method: To analyze the terms used in the international classification between 1979 and 2014, comparing literature and clinic experience.

Results: Disease classification nomenclature represents health information for constructing meanings regarding the origin and evolution of the disease and the therapeutic process. The term PI allows the medical view of the patient to be glimpsed. Under this condition, there is a dissociation between physical sleep and sleep as a psychological experience.

Conclusions: The new nomenclature (Paradoxical Insomnia) emphasizes the paradox that exists between objective and subjective data, but what needs to be considered is the patient's experience of psychological deprivation of sleep. We believe that the term "sleep state misperception" is more appropriate, since it is closer to the subjective experience of patients, taking into consideration the psychological suffering and the physical and mental wastage.
\end{abstract}

Keywords: Sleep State Misperception, Paradoxical Insomnia, Sleep Disorder, Neurology, Psychology.

\section{INTRODUCTION}

Among sleep disorders, Sleep State Misperception $(\mathrm{SSM})^{(1)}$ intrigues and challenges the specialists. Since its characterization as "insomnia with no objective findings", ${ }^{(2)}$ it has already been named "Subjective Insomnia" and "Pseudo-insomnia" and it was recently classified as "Paradoxical Insomnia" (PI). ${ }^{(3)}$ SSM is a disorder in which a complaint of insomnia is presented but without any objective clinical evidence for the presence of sleep disturbances. ${ }^{(1,3)}$ Polysomnography (PSG) on individuals with SSM presents few abnormalities. Attention is drawn greatly to the fact that normal or very little altered architecture and, especially, sleep efficiency are observed. These findings are at odds with the extreme insomnia that these patients report. They underestimate the total duration of sleep and overestimate their sleep latency. ${ }^{(3)}$

Even without objective evidence, the committees involved in producing sleep disorder classifications ${ }^{(1-3)}$ have included SSM in the insomnia group. This suggests that it is a sleep disorder, not only because of the patient's complaint but also because of the need for a differential diagnosis.

${ }^{1}$ Corresponding Author: luabarreto@ hotmail.com 


\section{American Research Journal of Medicine and Surgery, Volume 1, Issue 1, 2015}

ISSN 2379-8955

The aim of this work is to discuss the adoption of the term "paradoxical insomnia by the last international classification.

\section{METHODS}

We analyzed the terms used in the international classification between 1979 and 2014, comparing literature and the clinic experience on this disease.

\section{RESULTS}

In its first definition, a real situation was described: insomnia without objective findings. Subsequently, it was also presented as "pseudo-insomnia". In our view, this was a pejorative description, since it seems to suggest that the disorder has a false nature, or that the complaint of the patient suffering from SSM is false. On the other hand, the source of knowledge for the term "subjective insomnia" is in the complaint and this term gives indications that recognize the patient's experience and suffering. Much of the name-disease incongruence expressed in the literature and, particularly, in clinical practice is due to the word "insomnia". This word does not apply to individuals who actually sleep biologically and is not appropriate for the particular way that patients with SSM become ill. It is clear to clinicians that there is a dissociation between physical sleep, which is electrically detectable by means of technological equipment, and sleep as a psychological experience, which can only be recognized and expressed through patients' discourse or complaints. This divergence between objectivity and subjectivity shows a biopsychological dissociation in sleep. The latest classification with the new nomenclature (Paradoxical Insomnia), which was published a few years ago although, curiously, it has not been adopted in recent studies, aimed to emphasize the paradox that exists between the objective and subjective data. It gives preference to what the technology indicates and distances itself from clinical assessments and from what patients themselves might bring as contributions towards broader comprehension of this disorder.

Since the first classification almost thirty years ago, the name given to this disorder has kept on moving away from the experience of individuals who suffer from this condition and giving substance to the difficulty in finding a response to patients' complaints.

It is necessary to distinguish diagnosis from classification. Diagnosis means to recognize a clinical entity while classification is an attempt to organize different conditions within groups or categories that keep common relations. To make a diagnostic is to identify patients' morbid conditions as well to recognize specific experiences, singularities and conditions. A classification can be used as a clinical tool and it cannot be taken as a direct representation of a clinical condition or understood as a reified concept. ${ }^{(4)}$

In addition to being an attempt to classify the problem, conceptualization of a disease is also a means of discerning the relationship between the specialists, the disease and the patient. The fact that this sleep disorder has been classified while it has not been objectively confirmed would explain why the official designation of this disease has undergone transformations over the years, since these transformations reveal the search to make sense of and understand this disorder.

Studies on SSM are focused on its neurophysiological aspects. In addition, they discuss whether SSM should be considered to be a separate diagnostic condition, ${ }_{9}^{(5-7)}$ a subtype of primary insomnia, or just a prodromic symptom. ${ }^{(8-}$

One recent study ${ }^{(10)}$ proposed expanding the concept of misperception to "Positive Sleep State Misperception" (PSSM) for patients who overestimate their number of hours of sleep and "Negative Sleep State Misperception" (NSSM) for the classical syndrome in which patients underestimate their length of sleep. These researchers suggested that PSSM and NSSM were the extremes of a single spectrum into which patients with a normal perception of sleep would also fit. It is of interest to note that this new nomenclature was only cited in the introduction and abstract of the article. This study demonstrates how specialists may be neglecting clinical data and missing opportunities to explore and expand concepts and to discover new relationships between objective and subjective data.

Just as in cases of distortion of the body image, which is common in conditions of eating disorders, an intervention that is dedicated only towards the biological dimension, ignoring the psychosocial aspects, will not be complete and efficient. It is fundamentally important to have the patient's perspective in relation to his history, experiences and socio cultural world. The subjective notions of individuals with SSM need to be accessed, so as to integrate the psychological and physiological conscience, and to join this up with the socio historical context ${ }^{(11)}$. 


\section{American Research Journal of Medicine and Surgery, Volume 1, Issue 1, 2015}

ISSN 2379-8955

Determining patients' perceptions of the disease presupposes using qualitative examination: an interpretation of the pathological phenomenon from the experiences, picking up any variations and particular features ${ }^{(12)}$. Clinical assessment is not an instrument for discovering a truth that is still unknown but, rather, a certain way of displaying a truth that has already been found and presenting it so that it can be systematically revealed ${ }^{(12)}$.

In this respect, through clinical assessment, we can find the legitimate and authentic source from the experiences that patients narrate. After all, to grasp how the discourse is mutating, the language through which the ways of seeing and saying things are still expressed needs to be accessed, and attention needs to be directed to the "things" and "words" that have not yet become separated ${ }^{(12)}$.

We enter two areas of discussion that are joined together: the significance of the disease, along with how its significance is communicated, and the repercussions of this on the treatment and on the patient.

Is it sufficient to explain and demonstrate to patients that they are managing to sleep and that they do not need to be worried? Our clinical observations within Neuro-Sono Sleep Center Outpatient (UNIFESP) suggest that taking this attitude does not reduce patients' complaints ${ }^{(11)}$. From the physician's point of view, the objective data signal that the problem of the patient with the complaint lies within another area that is no longer within his sphere of influence. Nevertheless, we cannot turn our backs on such individuals and believe that the solution consists only of clarifying the results from these patients' tests or prescribing medications. Through doing this, either communication will not take place, or there will not be any meeting of minds in the discourse used. On the one hand, there is the medical explanation with evidence that the patient's complaint has no objective basis, and on the other hand, there is the subjective report from the patient who is suffering from insomnia, a particular type of insomnia that is invisible to all eyes except within his own world. This imposes an even greater burden on the patient, including within his family, where his account of inability to sleep is contested.

What emerges from this and needs to be considered is the patient's suffering and his experience of psychological deprivation of sleep, and we should intervene in this respect. If we find symptoms like somnolence, irritability, lack of concentration and headaches within the physiological deprivation of sleep, the psychological deprivation will also have effects on the patient's life, such as worrying about future physical and mental exhaustion, increased anxiety and complaints of memory loss.

\section{CONClusions}

The name Paradoxical Insomnia, listed in the latest classification, does not seem to represent the true clinical spectrum of this disease but, rather, a small collection of "paradoxes" going from severe sleep deprivation to mild or no functional impact. The term Paradoxical Insomnia allows the medical view of the patient to be glimpsed, and not what this disease may particularly represent.

The nomenclature used in the classification is not just a whim: it represents health information for constructing meanings regarding the origin and evolution of the disease and of the therapeutic process. For this reason, we believe that maintaining the term "Sleep State Misperception" is more appropriate, since it is closer to the subjective experience of patients who suffer from this condition, taking into consideration the psychological suffering and the physical and mental wastage.

\section{REFERENCES}

[1] ICSD-International Classification of Sleep Disorders: diagnostic and coding manual. Diagnostic Classification Steering Committee, Thorpy MJ, Chairman. Rochester, Minnesota: American Sleep Disorders Association; 1990.

[2] Association of Sleep Disorders Centers. Diagnostic Classification of sleep and arousal disorders. Sleep. 1979; 2:1-137.

[3] ICSD 2 - International Classification of Sleep Disorders 2nd ed.: diagnostic and coding manual. Diagnostic Classification Steering Committee, Thorpy MJ, Chairman. Rochester, Minnesota: American Sleep Disorders Association; 2005.

[4] Kendell R, Jablensky A. Distinguishing Between the Validity and Utility of Psychiatric Diagnoses. Am J Psychiatry. 2003; 160:4-12.

[5] Reynolds CF, Kupfer DJ, Buysse DJ, Coble PA, Yeager A. Subtyping DSM-III-R primary insomnia: a literature review by the DSM-IV work group on sleep disorders. Am J Psychiatry. 1991; 148:432-8.

[6] McCall WV, Edinger JD. Subjective total insomnia: an example of sleep state misperception. Sleep. 1992;15:71-3.

[7] Edinger JD, Krystal AD. Subtyping primary insomnia: is sleep state misperception a distinct clinical entity? Sleep Med Rev. 2003;7:201-2.

[8] Salin-Pascual RJ, Roehrs TA, Merlotti LA, Zorick F, Roth T. Long-term study of the sleep of insomnia patients with sleep state misperception and other insomnia patients. Am J Psychiatry. 1992;149:904-8. 
American Research Journal of Medicine and Surgery, Volume 1, Issue 1, 2015

ISSN 2379-8955

[9] Bonnet MH, Arand DL. Physiological activation in patients with sleep state misperception. Psychosom Med. 1997; 59:533-40.

[10] Trajanovic NN, Radivojevic V, Kaushansky Y, Shapiro CM.. Positive sleep state misperception: A new concept of sleep misperception. Sleep Med. 2007; 8:111-8.

[11] Barreto LA, Coin-Carvalho JE, Carvalho LBC, Prado LBF, Prado GF. Psychosocial Features of Brazilian Patients with Paradoxical Insomnia: a qualitative study. J Sleep Disor: Treat Care 2014, 3:3. http://dx.doi.org/10.4172/23259639.1000140

[12] Foucault M. The Birth of the Clinic: an archaeology of medical perception. New York: Random House; 1994. 